\title{
EvoDevo
}

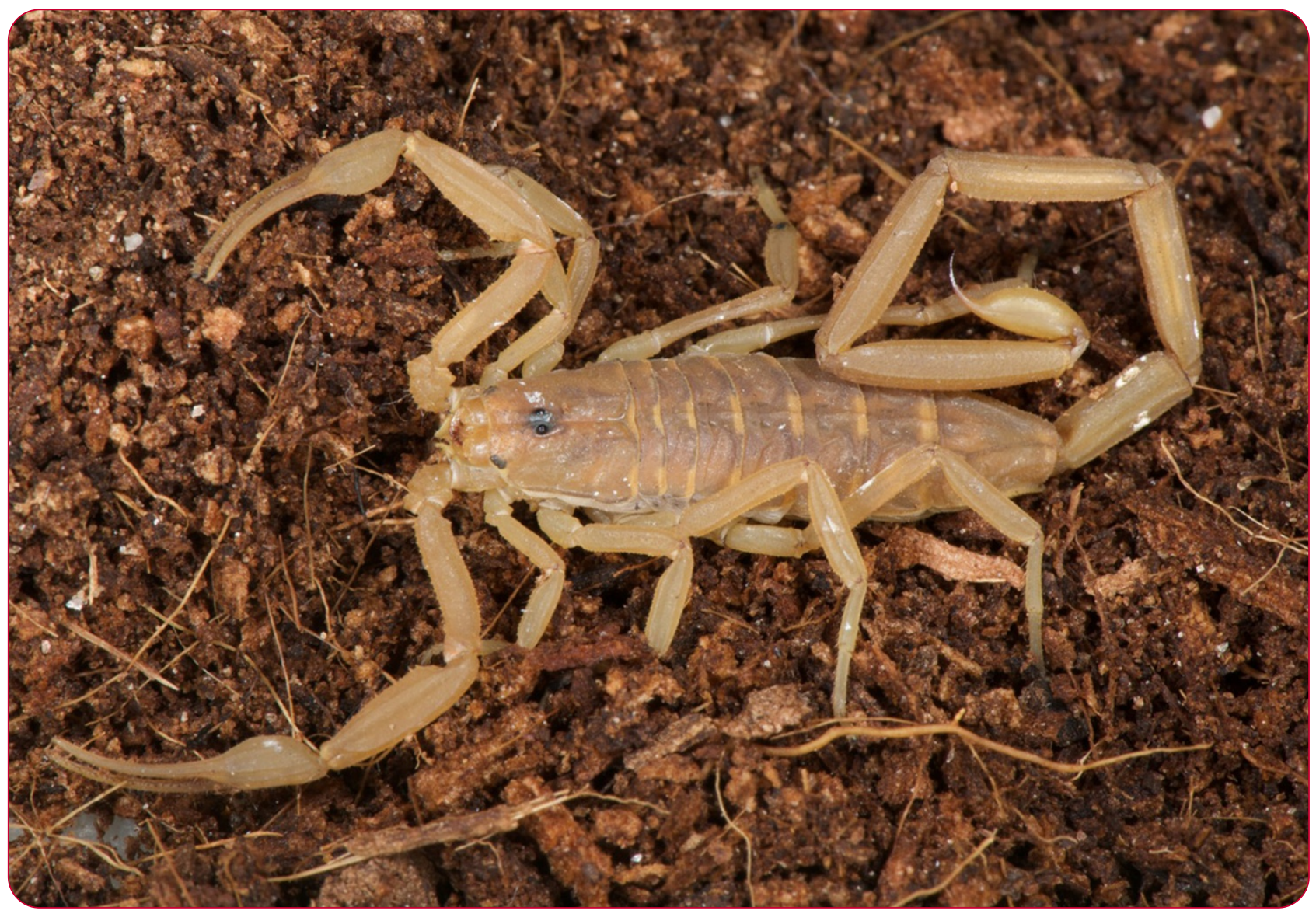

\section{Subdivision of arthropod cap-n-collar expression domains is restricted to Mandibulata}

Sharma et al. 


\title{
Subdivision of arthropod cap-n-collar expression domains is restricted to Mandibulata
}

\author{
Prashant P Sharma ${ }^{1 *}$, Tripti Gupta ${ }^{2}$, Evelyn E Schwager ${ }^{2}$, Ward C Wheeler ${ }^{1}$ and Cassandra G Extavour ${ }^{2}$
}

\begin{abstract}
Background: The monophyly of Mandibulata - the division of arthropods uniting pancrustaceans and myriapods - is consistent with several morphological characters, such as the presence of sensory appendages called antennae and the eponymous biting appendage, the mandible. Functional studies have demonstrated that the patterning of the mandible requires the activity of the Hox gene Deformed and the transcription factor cap-n-collar (cnc) in at least two holometabolous insects: the fruit fly Drosophila melanogaster and the beetle Tribolium castaneum. Expression patterns of cnc from two non-holometabolous insects and a millipede have suggested conservation of the labral and mandibular domains within Mandibulata. However, the activity of $\mathrm{cnc}$ is unknown in crustaceans and chelicerates, precluding understanding of a complete scenario for the evolution of patterning of this appendage within arthropods. To redress these lacunae, here we investigate the gene expression of the ortholog of cnc in Parhyale hawaiensis, a malacostracan crustacean, and two chelicerates: the harvestman Phalangium opilio, and the scorpion Centruroides sculpturatus.

Results: In the crustacean $P$. hawaiensis, the segmental expression of $P h-c n c$ is the same as that reported previously in hexapods and myriapods, with two distinct head domains in the labrum and the mandibular segment. In contrast, Po-cnc and Cs-cnc expression is not enriched in the labrum of either chelicerate, but instead is expressed at comparable levels in all appendages. In further contrast to mandibulate orthologs, the expression domain of Po-cnc posterior to the labrum is not confined within the expression domain of Po-Dfd.

Conclusions: Expression data from two chelicerate outgroup taxa suggest that the signature two-domain head expression pattern of $\mathrm{cnc}$ evolved at the base of Mandibulata. The observation of the archetypal labral and mandibular segment domains in a crustacean exemplar supports the synapomorphic nature of mandibulate cnc expression. The broader expression of Po-cnc with respect to Po-Dfd in chelicerates further suggests that the regulation of cnc by Dfd was also acquired at the base of Mandibulata. To test this hypothesis, future studies examining panarthropod cnc evolution should investigate expression of the cnc ortholog in arthropod outgroups, such as Onychophora and Tardigrada.
\end{abstract}

Keywords: Amphipod, cap-n-collar, Centruroides, Deformed, Harvestman, Labrum, Mandible, Parhyale, Phalangium, Scorpion

\section{Background}

Gene expression as evidence for phylogenetic relationships

As indicators of phylogenetic relationships, arthropod embryonic gene expression patterns are among the most idiosyncratic, frequently lending themselves to ambiguous statements of homology. This stems in part from limitations in taxonomic sampling; comparative gene expression data are presently available from approximately 25 arthropod

\footnotetext{
* Correspondence: psharma@amnh.org

'Division of Invertebrate Zoology, American Museum of Natural History,

Central Park West at 79th Street, New York, NY 10024, USA

Full list of author information is available at the end of the article
}

species [1-4], a minuscule fraction of those for which nucleotide sequence data have been collected. In addition, as evolutionary developmental biology is often driven by inquiry into the origins of particular morphological structures, the state of a gene's deployment is often not assessed in lineages and/or specific stages that lack a structure of interest, thereby engendering gaps in comparable expression data. As a consequence, the degree to which expression patterns are conserved is largely unknown for many well-characterized genes involved in embryogenesis, barring such exceptions as anterior Hox genes, segmentation genes, limb-patterning genes, and some neurogenetic markers [1,5-7].

\section{Biomed Central}


In other cases, even when broader taxonomic sampling increases confidence in assessments of the evolution of gene expression, the incidence of homoplasy (and particularly reversals) can create further ambiguity in the interpretation of evolutionary patterns. The sum of phylogenetic and phylogenomic studies of arthropods supports the monophyly of Mandibulata - a clade comprised of myriapods, a paraphyletic group commonly termed crustaceans, and hexapods - and its sister relationship to Chelicerata - a clade composed of arachnids, xiphosurans, and pycnogonids (Figure 1A) [8,9]. Morphological data are strongly consistent with these relationships: mandibulates are characterized by a six-segmented anterior tagma (the head) bearing antennae on the deutocerebral segment and no locomotory appendages, whereas the seven-segmented anterior tagma (the prosoma) of euchelicerates bears the namesake appendage on the deutocerebral segment, and all walking legs with a distinct podomere called the patella, which confers the appearance of a "double-knee". Contrary to this topology, the head-patterning gene collier ( $\mathrm{col}$ ) is strongly expressed in the intercalary segment of myriapods and hexapods, but not in the corresponding segments of crustaceans (the second antennal segment), chelicerates (the pedipalpal segment), or onychophorans - the sister lineage of arthropods [10-12]. These data have been interpreted to mean a possible role for $\mathrm{col}$ in patterning the appendage-less tritocerebral state of hexapods [10]. Indeed, the perfect correspondence between the incidence of the intercalary segment and the expression domain of $\mathrm{col}$ is suggestive of a convergent function for $\mathrm{col}$ in patterning an appendage-less segment. However, an alternative interpretation of these data has been putative support for the Atelocerata hypothesis, which unites Myriapoda and Hexapoda as sister groups (Figure 1B) [11]. This interpretation is implicitly based on Dollo parsimony (non-reversibility of a given character state) and renders a large number of better-sampled morphological and molecular characters homoplastic [13]. A comparable example is the case of leg gap gene expression, where the expression boundaries of homothorax (hth) and extradenticle (exd) support the controversial sister relationship of Myriapoda and Chelicerata (Figure 1C) $([7,14]$, but see [15]), a relationship otherwise only poorly supported by some analyses of molecular sequence data $[16,17]$.

A counterexample of gene expression evolution consistent with the total evidence phylogenetic tree may be provided by examining an unambiguous synapomorphy

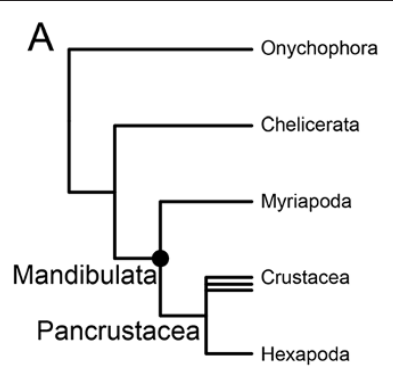

MANDIBULATA

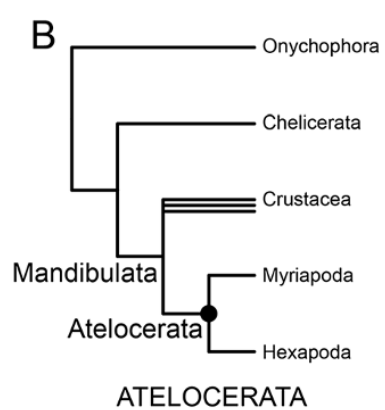

ATELOCERATA

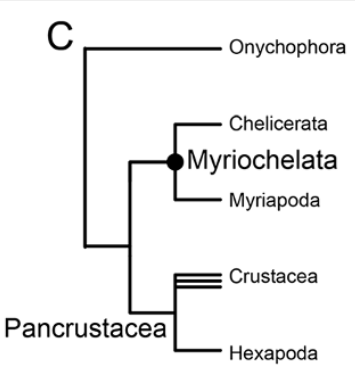

MYRIOCHELATA

D

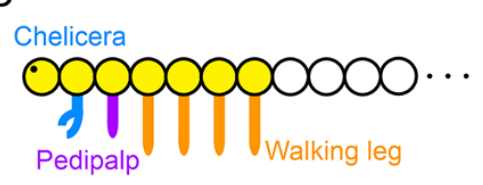

CHELICERATA

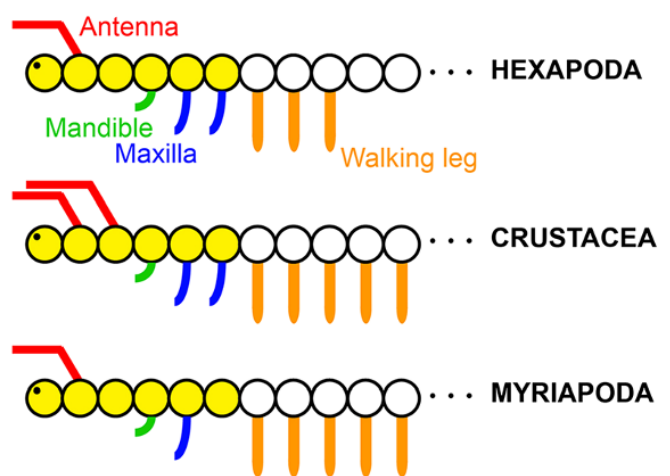

Figure 1 Competing hypotheses in arthropod phylogeny. (A) Mandibulata unites the non-chelicerate arthropods in a clade and is the hypothesis most stably recovered in phylogenetic analyses. Multiple terminal icons indicate the non-monophyly of Crustacea. (B) The Atelocerata hypothesis unites hexapods and myriapods to the exclusion of crustaceans. (C) Myriochelata unites the myriapods and chelicerates in a clade. (D) Segmental architecture of Arthropoda. The depiction of Myriapoda corresponds to the millipede bauplan (a second maxilla is present in other myriapod lineages). The anterior-most head segment, or protocerebral segment, is to the left. Yellow circles indicate the anterior tagmata, the chelicerate prosoma and the mandibulate head. 
of Mandibulata: the mandible. The eponymous biting appendage of mandibulates is a gnathobasic structure occurring on the fourth head segment of all mandibulates, irrespective of the architecture of the remaining head segments (Figure 1D) [18-20]. Two genes are required for the proper formation of the mandible: the Hox gene Deformed $(D f d)$ and the basic leucine zipper family transcription factor cap-n-collar (cnc) [21-23]. In the fruit fly Drosophila melanogaster, $D f d$ is required for patterning the mandibular and first maxillary segments [24]. In both D. melanogaster and the beetle Tribolium castaneum, cnc is expressed in two domains, the first in the labrum and the second in the mandibular segment [25]. Functional studies in both species have shown that $c n c$ is required for formation of the labrum, and for differentiating the mandible from the maxilla. A loss-of-function mutation in D. melanogaster results in ectopic maxillary structures on the mandibular segment (for example, hooks and cirri), and RNA interference-mediated knockdown in T. castaneum in complete mandible-to-maxilla homeotic transformation [21-23,25]. In both insects, $c n c$ downregulates the expression of $D f d$ in the mandibular segment over the course of mandibular limb bud growth. Intriguingly, $c n c$ is activated by $D f d$ in T. castaneum but not in D. melanogaster [25]. The polarity of this regulation with respect to phylogeny is not known.

Expression data for orthologs of $c n c$ are available for a hemimetabolous insect (Oncopeltus fasciatus), a non-metamorphic insect (Thermobia domestica) and a millipede (Glomeris marginata), all of which bear a characteristic labral and mandibular domain [12,26,27]. Intriguingly, the mandibular domains of all mandibulate cnc orthologs occur within the $D f d$ domains of these lineages, and a downregulation of $D f d$ in the mandibular segment of older stage embryos has been observed across mandibulates as well. These conserved expression dynamics have been used to suggest that the mandible-patterning function of $c n c$ evolved at the base of Mandibulata within the domain of $D f d$ [25].

However, as demonstrated by the case of $\mathrm{col}$, $h \mathrm{th}$, and exd, many embryonic genes are prone to convergence and/or reversals. Inasmuch as cnc expression is unknown in crustaceans and non-mandibulate arthropods, the assignation of the two-domain head expression pattern to the ancestor of Mandibulata remains ambiguous. To refine the inference of evolution of $c n c$ expression and its regulation by $D f d$, we investigated the expression of $c n c$ in the malacostracan crustacean Parhyale hawaiensis and two chelicerates: the harvestman Phalangium opilio and the scorpion Centruroides sculpturatus. We used these data to test the prediction that the two-domain head expression pattern is conserved in the crustacean, whereas an unknown, non-mandibulate state occurs in the chelicerates.

\section{Methods}

\section{Embryo cultivation and fixation}

$P$. hawaiensis adults were cultured in artificial seawater (Instant Ocean, Blacksburg, VA, USA) with crushed coral at $28^{\circ} \mathrm{C}$. Animals were fed daily with ground aquaculture feed: $40 \%$ TetraPond $^{\circ}$ wheat germ sticks, $40 \%$ TetraMin $^{\odot}$ flake food, and $20 \%$ Tropical $^{\bullet}$ spirulina (Tetra, Blacksburg, VA, USA). Gravid females were anesthetized with $\mathrm{CO}_{2}$, and embryos were collected as described previously [28]. Embryos were fixed for in situ hybridization by incubating in $3.7 \%$ formaldehyde in $1 \times$ PBS for 2 minutes at $75^{\circ} \mathrm{C}$ followed by 20 minutes in $3.7 \%$ formaldehyde in $1 \times$ PBS at $4^{\circ} \mathrm{C}$. Membranes were manually dissected from embryos in PBS and embryos fixed overnight at $4^{\circ} \mathrm{C}$.

Adults of the harvestman $P$. opilio were hand collected between 21.00 and 03.00 from Weston, Massachusetts, USA in May through July, 2013. Housing, feeding, embryo cultivation, and embryo fixation followed published protocols [29].

Adult females of the scorpion C. sculpturatus were purchased from an animal supplier (Hatari Invertebrates, AZ, USA). Females were anesthetized with $\mathrm{CO}_{2}$ and embryos dissected from the ovary following a modification of a published protocol [30]. Embryos were dissected to remove yolk and fixed in $3.7 \%$ formaldehyde in $1 \times$ PBS at room temperature overnight.

Gene identification and whole mount in situ hybridization Potential orthologs of $c n c$ were identified in the annotated developmental transcriptomes of $P$. hawaiensis (deposited in the ASGARD Project database; [31]), P. opilio (Sharma and Giribet, unpublished data), and C. sculpturatus (Sharma and Wheeler, unpublished data). For C. sculpturatus, an ortholog of the Hox gene Antennapedia was additionally identified and used as a positive control for the cnc in situ hybridization experiments. Gene identity of $c n c$ orthologs was confirmed by BLAST and alignments generated from conceptual peptide translations (Additional file 1: Figure S1). Sequences of all genes are deposited in GenBank.

Templates for riboprobe synthesis for P. hawaiensis and P. opilio were generated following a published protocol [32]: genes were amplified by PCR using gene-specific primers (GSPs) with an added linker sequence (5' ggccgcgg- 3 ' for the forward primer end and 5 '-cccggggc- 3 ' for the reverse primer). A T7 polymerase binding site for anti-sense or sense probe synthesis was generated in a second PCR using the forward or reverse GSP and a universal primer binding to the $3^{\prime}$ or $5^{\prime}$ linker sequence with an added T7 binding site, respectively. GSPs were designed from the corresponding transcriptomic assemblies. For P. opilio, two pairs of sense and anti-sense probes with only partial overlap over the basic region leucine zipper domain were generated to establish the validity of the expression data. Templates for riboprobe synthesis for 
C. vittatus were generated by PCR-amplified GSPs, and cloning amplicons using the $\mathrm{TOPO}^{\circ} \mathrm{TA}^{\mathrm{T}} \mathrm{Cloning}^{\circ}$ Kit with One Shot $^{\circ}$ Top10 chemically competent Escherichia coli (Invitrogen, Carlsbad, CA, USA), following the manufacturer's protocol. Amplicon identities were verified by direct sequencing. A list of the GSPs used for generating sense and anti-sense probes is provided in Additional file 2: Table S1.

Whole mount in situ hybridization on $P$. hawaiensis embryos was performed as described previously [33] with the following modifications: prior to rehydration, embryos were cleared by incubation in xylene for 20 minutes. Hybridization was performed at $67^{\circ} \mathrm{C}$. Following post-fixation, embryos were incubated in detergent solution (1.0\% SDS, $0.5 \%$ Tween, $50.0 \mathrm{mM}$ Tris- $\mathrm{HCl}$ (pH 7.5), $1.0 \mathrm{mM}$ EDTA (pH 8.0), $150.0 \mathrm{mM}$ $\mathrm{NaCl}$ ) for 30 minutes and then fixed again in $3.7 \%$ formaldehyde for 30 minutes. After hybridization, embryos were washed twice in $2 \times$ saline sodium citrate for 30 minutes and then twice in $0.2 \times$ saline sodium citrate for 30 minutes. Probes were visualized using nitro-blue tetrazolium and 5-bromo-4-chloro-3'-indolyphosphate staining reactions, run overnight at $4^{\circ} \mathrm{C}$.

In situ hybridization for $P$. opilio followed published protocols [29]. For C. sculpturatus, in situ hybridization followed the same protocol as for P. opilio. Staining reactions for detection of transcripts lasted between 0.5 and 6 hours at room temperature. Embryos were subsequently rinsed with $1 \times \mathrm{PBS}+$ Tween-20 $0.1 \%$ to stop the reaction, counterstained with Hoechst 33342 (Sigma-Aldrich, St. Louis, MO, USA) $10 \mu \mathrm{g} / \mathrm{ml}$ to label nuclei, post-fixed in $4 \%$ formaldehyde, and stored at $4^{\circ} \mathrm{C}$ in glycerol. Embryos were mounted in glycerol and images were captured using an $\mathrm{HrC}$ AxioCam and a fluorescence zoom stereomicroscope driven by Zen (Zeiss, Oberkochen, Germany).

\section{Results}

\section{Identification of cnc orthologs}

Putative single-copy $c n c$ orthologs between 393 bp and $739 \mathrm{bp}$ in length were identified in the transcriptomes of all three species. To confirm gene orthology, multiple sequence alignment of $c n c$ amino acid sequences was conducted using MUSCLE v. 3.6 [34], comparing crustacean and chelicerate sequences to those of $D$. melanogaster, T. castaneum, and G. marginata. The conserved region of the alignment is shown in Figure 2; the complete alignment is provided in Additional file 1: Figure S1. Next, we studied gene expression in the embryos of $P$. hawaiensis and both chelicerates. As negative controls, we tested for expression of sense probes. In all cases, no staining was observed in sense controls (Additional file 3: Figure S2).

\section{Expression of $c n c$ in the crustacean $P$. hawaiensis}

Consistent with expression of $c n c$ in other mandibulates, $\mathrm{Ph}$-chc is expressed in disjunct head domains in limb bud stage embryos (stages 18-22). Expression in earlier stages occurs in the mandibular segment (stages 15-18; Figure $3 \mathrm{~A}, \mathrm{~B}$ ), and expands into labrum and the stomodeal wall as the mandibles elongate (stages 19-20; Figure 3C,D). By stage 20, the anterior-most domain comprises strong expression in labrum and the tissues around the stomodeum, forming a ring. This domain does not extend into the head lobes. The mandibular domain consists of strong expression in the mandibular limb buds. By stage 20, an additional expression domain is observed at the posterior terminus of the embryo, in a ring around the proctodeum (Figure 3D).

\section{Expression of $c n c$ in the harvestman $P$. opilio}

In early stages (stage 11), Po-cnc is expressed in the labrum, as well as all limb buds and the posterior end (Figure 4A,B). No expression is observed along the ventral midline of the prosomal segments. In older stages (stage 15), Po-cnc continues to be expressed in the labrum and all prosomal appendages, as well as outgrowing endites of the pedipalpal and L1 segments (Figure 4C). Comparably to $P$. hawaiensis, the labral domain extends into a ring of expression surrounding the stomodeum. Expression is observed in the eye fields as well as the posterior terminus. Identical expression patterns were obtained with either of two partially overlapping anti-sense probes, and no expression was observed with either complementary sense probe (Additional file 3: Figure S2; Additional file 4: Figure S3A-C).

\section{Expression of Antp and cnc in the scorpion C. sculpturatus}

There are currently no well-established laboratory scorpion model species, and due to the peculiar life history traits of scorpions (including live birth, small broods, gestation periods lasting multiple months), collecting embryos is largely a matter of chance. Obtaining specific developmental stages is thus a matter of intensive sampling of adult females. In the present study, we obtained embryonic stages of C. sculpturatus comparable to stage 15 of $P$. opilio (Figure 4C,D), as inferred from (1) completion of appendage podomerization, (2) formation of gnathobases, and (3) completion of opisthosomal segment addition.

In order to establish the validity of the in situ hybridization protocol for this species, gene expression of the Antp ortholog was additionally investigated. We reasoned that the conservation of the Antp expression domain in multiple chelicerate species $[1,29]$ would make this gene an appropriate choice as a positive control. Using a Cs-Antp anti-sense probe, we found that, as in all known chelicerates, the anterior expression boundary of 


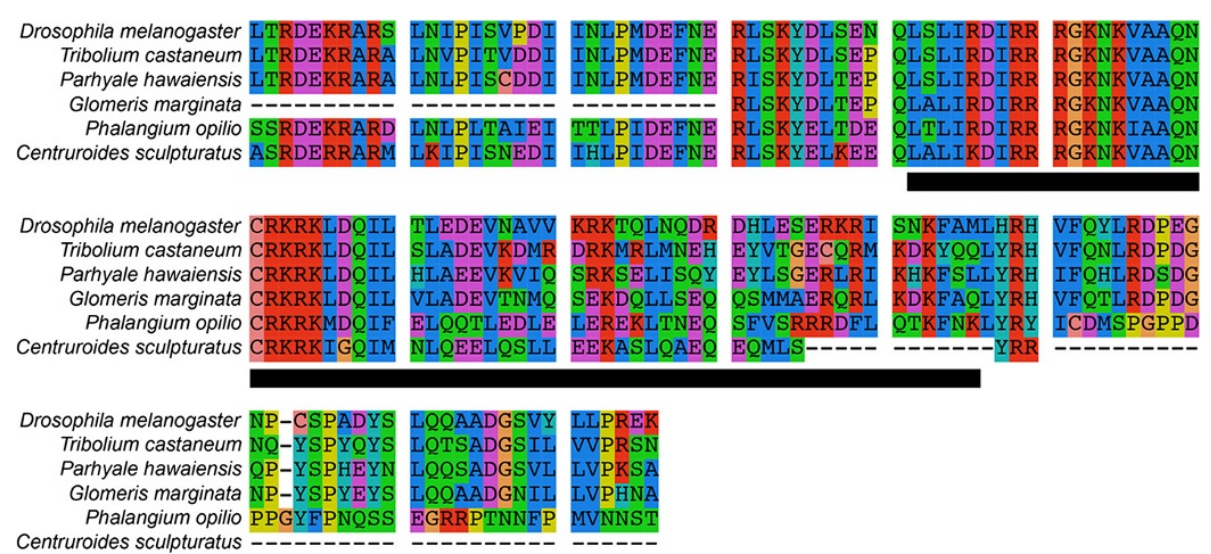

Figure 2 Multiple sequence alignment of arthropod cap-n-collar orthologs. The conserved region comprising 146 amino acids is shown. Black bar indicates the basic region leucine zipper domain.

Cs-Antp occurs in the posterior part of the L4 segment. Cs-Antp is expressed throughout the posterior tagmata (mesosoma and metasoma). Complete absence of staining is observed in the prosoma (Additional file 4: Figure S3D) and in sense controls (not shown), suggesting that our in situ protocol can effectively distinguish signal from background.

Using this in situ hybridization protocol, we found that Cs-cnc is expressed throughout the prosoma, including in the eye fields, the labrum, the appendages, the coxapophyses, and the ventral ectoderm (Figure 4D). Cs-cnc is additionally expressed in the mesosomal ventral ectoderm, the pectines, the book lungs, and throughout the metasoma. Expression is weakest in the periphery of the O4-O7 segments, which bear the book lungs.

\section{Discussion}

Beyond testing a particular evolutionary scenario through repeated observation of a putatively conserved trait, extensive sampling of lineages for a character of interest is essential for identifying the origins of evolutionary novelties, such as the arthropod mandible. Here we investigated the evolution of $c n c$ expression and tested the association of $c n c$ domains with mandibular patterning. Given that published expression data are available only for four insects and one myriapod [21-27], we aimed to corroborate the conservation of $c n c$ domains for the first
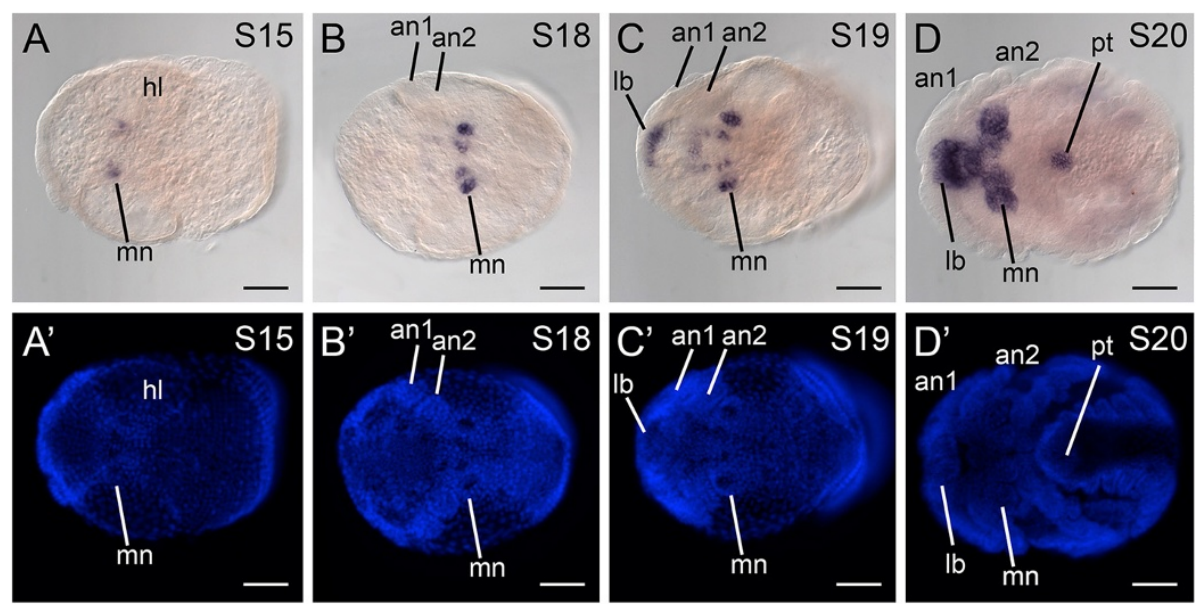

Figure 3 Parhyale hawaiensis cap-n-collar ortholog is expressed as in other mandibulates. (A) Stage 15 embryo in ventral view. Expression of Ph-chc is detected in the mandibular anlagen. (B) Stage 18 embryo in ventral view, showing expression of Ph-cnc in the mandibular limb buds. (C) Stage 19 embryo in ventral view. Expression of Ph-cnc occurs in discrete domains in the labrum, the mandibles, and in the ventral ectoderm of the mandibular segment. (D) Stage 20 embryo in ventral view. Expression of Ph-cnc encompasses the labrum, the stomodeal wall, the

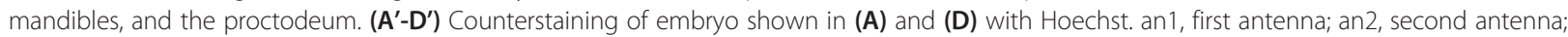
Ib, labrum; mn, mandible; pt, proctodeum. Scale bars for all figures are $100 \mu \mathrm{m}$. 


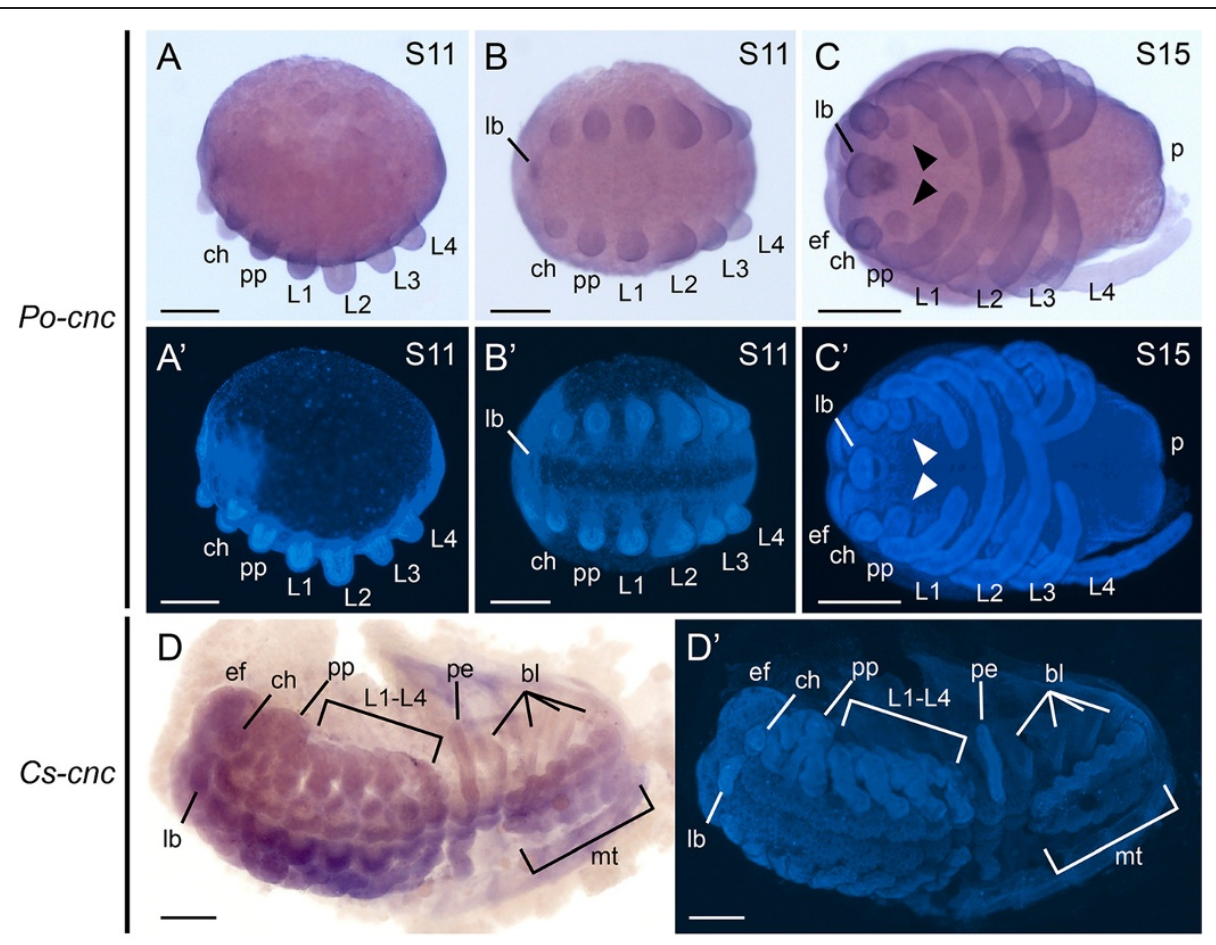

Figure 4 Chelicerate cap-n-collar orthologs are expressed throughout the germ band. (A) Stage 11 embryo of the harvestman Phalangium opilio in lateral view. Po-cnc (488-bp anti-sense probe) is expressed throughout the germ band, including in all prosomal appendages. (B) Same embryo as in (A) in ventral view. (C) Stage 15 embryo of P. opilio in ventral view. Po-cnc continues to be expressed throughout the germ band. Expression is additionally detected in the coxapophyses (arrowheads). (D) Expression of Cs-cnc in the scorpion embryo. Cs-cnc is detected throughout the germ band, including in the eye fields, all prosomal and opisthosomal appendages, and in the metasoma (tail). ( $\mathbf{A}^{\prime}$-'D') Counterstaining of embryos shown in (A-D) with Hoechst. bl, book lung; ch, chelicera; ef, eye field; lb, labrum; L1-L4, leg 1-leg 4; mt, metasoma; p, posterior end; pe, pectine; pp, pedipalp. Scale bars are $200 \mu \mathrm{m}$ for (A-C) and $500 \mu \mathrm{m}$ for (D).

time in a crustacean species, and infer their origin by examining $c n c$ expression in the sister group of mandibulates, the chelicerates.

\section{Ph-cnc expression supports the archetypal mandibulate pattern}

The localization of $P h$-cnc transcripts in the labrum and mandibular segments of the malacostracan $P$. hawaiensis - the "cap" and "collar" domains, respectively supports this characteristic expression pattern as conserved among mandibulates. The restriction of the posterior head domain within the mandibular segment, in concert with the known function of $c n c$ in patterning mandibular identity in both $D$. melanogaster and T. castaneum, suggests conservation of $c n c$ function among mandibulates with respect to mandibular patterning. Similarly, conserved expression of $c n c$ in the labrum of all sampled mandibulates suggests that $c n c$ is required for the development of this structure; in D. melanogaster and T. castaneum, loss-of-function of $c n c$ results in the deletion of the labrum $[21,25]$.

One $c n c$ expression domain of unknown function in mandibulates is expression in the posterior-most segments.
In $P$. hawaiensis, $P h$-cnc is expressed in a ring of tissue surrounding the proctodeum (Figure 3D). Such a posterior expression domain occurs variably among insects; in $D$. melanogaster, T. castaneum, and O. fasciatus, cnc is not expressed in the posterior-most segments [21,25,27], whereas in the firebrat $T$. domesticus, $T d$-cnc is expressed from the A6 segment to the posterior terminus [26]. The functional significance of the posterior domain is not known, but may represent an evolutionary remnant of the unrestricted $c n c$ domain in the non-mandibulate arthropods.

\section{Chelicerate ortholog expression suggests subdivision of} cnc domains in the mandibulate ancestor

The conservation of the disjunct head expression domains of $c n c$ among the mandibulates precludes assessment of their evolutionary origin based on mandibulate data alone. To assess the evolution of the genetic network that may have precipitated the patterning of the mandible, we examined $c n c$ expression in two chelicerates, the harvestman and the scorpion. As inferred from Hox gene data, specifically the anterior boundary of $D f d$, the mandibular segment corresponds to the first walking leg segment in chelicerates (Figure 5) [1]. Given that more 


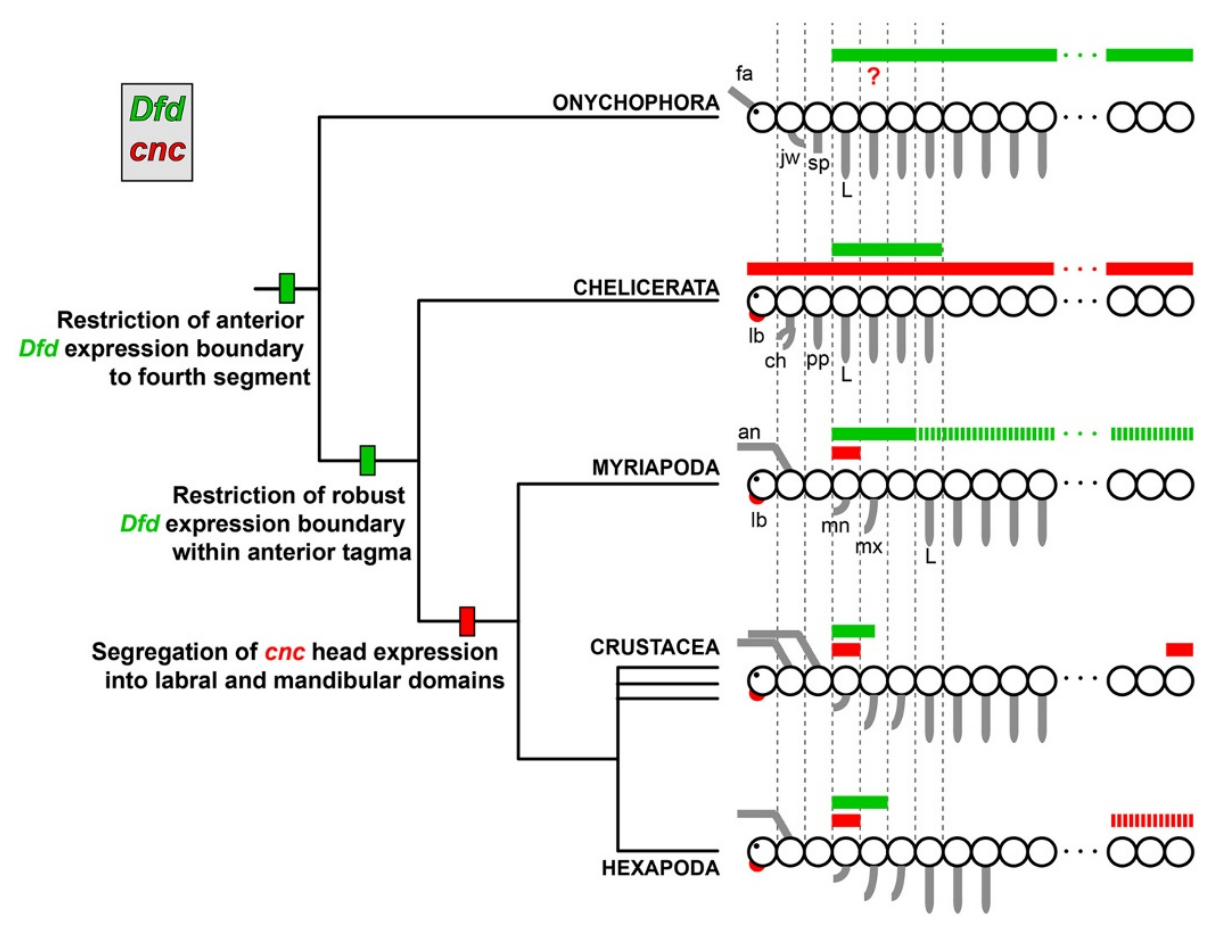

Figure 5 Inferred evolution of cap-n-collar and Deformed in Arthropoda. Known expression patterns of chc (red) and Dfd (green) from panarthropods suggest restriction of a robust Dfd domain to within an anterior tagma in the ancestor of Arthropoda. Hashed bars for myriapods indicate weak expression of Dfd in the trunk segments of the millipede (but not the centipede). Whereas cnc expression occurs throughout the developing chelicerate embryo, disjunct domains of cnc expression are exclusive to the mandibulates, thereby constituting a putative mandibulate synapomorphy. Hashed bars in the posterior terminus for hexapods indicate presence of $c n c$ in the posterior segments of some insects. ch, chelicera; fa, frontal appendage; jw, jaw; sp, slime papillae; L, leg; lb, labrum; mn, mandible; mx, maxilla.

anterior appendages are used for feeding in Chelicerata, we hypothesized that $c n c$ chelicerate expression would occur as a non-mandibulate but unknown state.

Consistent with this hypothesis, gene expression of $c n c$ orthologs in both the harvestman and the scorpion indicate nearly ubiquitous expression in examined developmental stages (Figure 4C,D; compare to Figure 3). In the early stages sampled for $P$. opilio, expression is observed throughout the germ band (Figure 4A,B). Po-cnc continues to be ubiquitously expressed throughout both the prosoma and opisthosoma at the developmental stage when the appendages are fully podomerized and elongate (stage 15) (Figure 4C). While we were unable to examine early limb bud stages of scorpions (prior to completion of opisthosomal segmentation), we observed a similar expression pattern in scorpion developmental stages with fully podomerized appendages and morphologically distinct opisthosomal organs (pectines and book lungs) (Figure 4D).

The function of $c n c$ in Chelicerata was not examined here, due to the lack of functional tools in the scorpion and the limited seasonality of the harvestman. Beyond arthropods, the functions of orthologs of $c n c$ have been studied in the nematode Caenorhabditis elegans and in vertebrates. In C. elegans, the ortholog of $c n c, s k n-1$, is required for the specification of ventral blastomere identity at the four-cell stage. In $s k n-1$ mutants the EMS blastomere, which normally forms pharyngeal and intestinal cells, acquires P2 cell identity and forms body wall muscle and hypodermal cells [35]. Nrf2, a vertebrate $c n c$ ortholog, has been implicated in oxidative stress response in mammals [36,37], a non-developmental role similarly observed in xenobiotic response in Drosophila [38]. These disparate functional data are suggestive of multiple co-options of $c n c$ throughout Bilateria to achieve various functions and preclude speculation on the role of $c n c$ in chelicerates.

The ubiquitous expression of chelicerate $c n c$ expression suggests that the expression and function of $c n c$ in distinct head appendage domains is exclusive to Mandibulata and presumably evolved in the ancestor of mandibulates. Alternatively, an equally parsimonious reconstruction could be evolution of subdivided $c n c$ domain at the base of Panarthropoda, and subsequent secondary evolution of the chelicerate state of $c n c$ expression. Under this hypothetical scenario, the $c n c$ ortholog of Onychophora would be predicted to have an expression domain comparable to that of Mandibulata.

However, we consider a shared expression pattern in Mandibulata and Onychophora unlikely for several 
reasons. First, like Chelicerata, Onychophora lack a mandible. Second, the first walking leg segments of both onychophorans and chelicerates are putatively homologous to each other, and to the mandibular segment of Mandibulata; only the first three head segments of Onychophora and Chelicerata have identities distinct from the walking legs, in contrast to the six-segmented mandibulate head (Figure 5). For these reasons, we consider a shared state between Onychophora and Chelicerata plausible. Nevertheless, assignation of $c n c$ subdivision to the base of Mandibulata remains ambiguous, and it is imperative to investigate $c n c$ expression in onychophorans and tardigrades to test this putative synapomorphy of mandibulates in future studies.

\section{Regulation of Dfd by cnc may have evolved within Mandibulata}

In all presently sampled branches of the mandibulate tree (hexapods, malacostracan crustaceans, and myriapods), part of $c n c$ expression is restricted to within the $D f d$ expression domain. In hexapods, the expression domain of $D f d$ spans the mandibular and maxillary segments (Figure 5). cnc arises within the $D f d$ domain and progressively downregulates $D f d$, with declining levels of $D f d$ expression signal in the mandibular segment over time [25]. Intriguingly, the temporal expression of $D f d$ follows the same pattern in the millipede G. marginata, with loss of expression in the distal mandible in older stages (Figure 4A-C of [39]). A similar expression pattern has been reported in the mandibular segment of the centipede Lithobius atkinsoni, namely the absence of $D f d$ expression in the distal mandible (note that the posterior boundary of $D f d$ is not the same in the two species; weak expression of $D f d$ is observed in the millipede trunk, but not in the centipede) (Figure 4C of [40]). These observations suggest conservation of the regulation of $D f d$ by $c n c$ in the mandibular segment of non-hexapod mandibulates. Unfortunately, functional tools are currently lacking in myriapods, precluding a direct test of this genetic interaction in either centipedes or millipedes.

In contrast to mandibulates, known $D f d$ expression in euchelicerates with eight-legged embryos (that is, all chelicerates except Pycnogonida, Acariformes, Parasitiformes, and Ricinulei) is restricted to the four walking leg segments, and does not wane in expression strength in the course of development $[29,41,42]$. Moreover, the occurrence of $c n c$ transcripts throughout the embryo, rather than exclusively within the chelicerate $D f d$ domain, disfavors regulation of cnc by $D f d$ in a manner comparable to the mandibulates' regulatory apparatus (Figure 5). These data suggest that the downregulation of $D f d$ within a specific $c n c$ domain constitutes a synapomorphy of Mandibulata that is required for the patterning of the mandible.

The interrelated evolution of $c n c$ and $D f d$ may be investigated in future by characterizing the expression domain of $c n c$ in onychophorans. Previous description of onychophoran Hox gene expression domains has reported broad expression of labial $(l b)$, proboscipedia $(p b), H o x 3$, and $D f d$ transcripts, from anterior boundaries shared with arthropods extending to the posterior terminus of the velvet worm embryo [43]. It has previously been suggested that the restriction of the posterior expression boundaries of Hox genes in arthropods precipitated the evolution of various tagmata (Figure 5) [1,43]. Ubiquitous expression of onychophoran $c n c$, comparable to expression of $c n c$ orthologs in chelicerates, would lend support to the evolutionary inferences made herein.

We suggest that future studies endeavoring to investigate mandible evolution should focus on two avenues of research: (1) developing functional tools in a species of Myriapoda to interrogate the regulatory dynamic of $c n c$ and $D f d$ in a basally branching mandibulate, and (2) identifying the function of $c n c$ in chelicerates. While several aforementioned aspects of scorpion life history will delay the development of functional tools in C. sculpturatus, RNA interference has proven successful in spiders, mites and, most recently, harvestmen [44-46].

\section{Conclusion}

The evolution of the mandible, an arthropod evolutionary novelty, has previously been linked to the function of $c n c$, and conserved expression of $c n c$ orthologs was heretofore observed in insects and a millipede. Here we investigated the expression of $c n c$ in a malacostracan crustacean and two chelicerates. We show that $c n c$ expression is conserved in all branches of the mandibulate phylogeny. By contrast, chelicerate $c n c$ is ubiquitously expressed in examined developmental stages, suggesting that evolution of the mandible may have involved the progressive subdivision of the $c n c$ expression domain.

\section{Additional files}

Additional file 1: Figure S1. Complete multiple sequence alignment of cap-n-collar orthologs.

Additional file 2: Table S1. Gene-specific primers used for synthesizing cap-n-collar probes.

Additional file 3: Figure S2. Chelicerate cap- $n$-collar sense probes. (A) Stage 13 embryo of Phalangium opilio stained with 488-bp sense probe in same solution and for the same period of time as embryos shown in Figure 4A-C. (B) Stage 14 embryo of Phalangium opilio stained with 739-bp sense probe in same solution and for the same period of time as embryos shown in Additional file 4: Figure S3B-C. (C) Centruroides sculpturatus embryo stained in same solution and for the same period of time as embryo shown in Figure 4D. $\left(A^{\prime}-C^{\prime}\right)$ Counterstaining of embryos shown in $(A-C)$ with Hoechst. Scale bars are $200 \mu \mathrm{m}$ for (A, B) and $500 \mu \mathrm{m}$ for (C).

Additional file 4: Figure S3. Additional controls for chelicerate cap-ncollar in situ hybridization experiments. (A) Design of partially overlapping probes for Po-cnc. (B) Stage 14 embryo of Phalangium opilio in ventral view, stained with 739-bp anti-sense probe. (C) Stage 16 embryo of Phalangium opilio in ventral view, stained with 739-bp anti-sense probe. (D) Expression of Cs-Antp, a positive control for Centruroides sculpturatus. As in all chelicerates for 
which Antp expression data are available, Cs-Antp is expressed from the posterior part of the $L 4$ segment to the posterior terminus. Dotted line indicates prosomal-mesosomal boundary. (D') Counterstaining of embryos shown in (D) with Hoechst. ch, chelicera; ef, eye field; L, leg; lb, labrum; me: mesosoma; mt, metasoma; pp, pp, pedipalp; pr: prosoma. Scale bar are $200 \mu \mathrm{m}$ for $(B, C)$ and $500 \mu \mathrm{m}$ for (D).

\section{Abbreviations}

BLAST: Basic local alignment search tool; bp: Base pair; GSP: Gene-specific primers; PBS: Phosphate buffered saline; PCR: Polymerase chain reaction.

\section{Competing interests}

The authors declare that they have no competing interests.

\section{Authors' contributions}

PPS conceived of the study. PPS and WCW generated the scorpion developmental transcriptome. CGE generated the crustacean developmental transcriptome and provided funding for part of the study. TG collected and analyzed the crustacean data; EES and PPS collected chelicerate data. EES, PPS, and TG analyzed expression patterns and drafted the manuscript All authors edited the manuscript and approved the final content for submission.

\section{Acknowledgements}

We are indebted to Roger D Farley for his detailed protocols for scorpion embryonic dissections and his encouragement to pursue study of scorpion development. Douglas Richardson facilitated imaging at the Harvard Center for Biological Imaging. The cover image was photographed by Gonzalo Giribet. PPS was supported by the National Science Foundation Postdoctoral Research Fellowship in Biology under Grant No. DBI-1202751. This work was partially supported by NSF grant IOS-1257217 to CGE and internal AMNH funds to WCW. Maximilian J Telford and three anonymous reviewers improved an earlier draft of the manuscript.

\section{Author details}

${ }^{1}$ Division of Invertebrate Zoology, American Museum of Natural History, Central Park West at 79th Street, New York, NY 10024, USA. ²Department of Organismic and Evolutionary Biology, Harvard University, 26 Oxford Street, Cambridge, MA 02138, USA.

Received: 3 September 2013 Accepted: 14 November 2013 Published: 9 January 2014

\section{References}

1. Hughes $C L$, Kaufman TC: Hox genes and the evolution of the arthropod body plan. Evol Dev 2002, 4:459-499.

2. Prpic NM, Tautz D: The expression of the proximodistal axis patterning genes Distal-less and dachshund in the appendages of Glomeris marginata (Myriapoda: Diplopoda) suggests a special role of these genes in patterning the head appendages. Dev Biol 2003, 260:97-112.

3. Simonnet F, Deutsch J, Quéinnec E: Hedgehog is a segment polarity gene in a crustacean and a chelicerate. Dev Genes Evol 2004, 214:537-545.

4. Abzhanov A, Extavour CG, Groover A, Hodges SA, Hoekstra H, Kramer EM, Monteiro A: Are we there yet? Tracking the development of new model systems. Trends Genet 2008, 24:353-360.

5. Damen WGM, Hausdorf M, Seyfarth E-A, Tautz D: A conserved mode of head segmentation in arthropods revealed by the expression pattern of Hox genes in a spider. Proc Natl Acad Sci USA 1998, 95:10665-10670.

6. Stollewerk A, Simpson P: Evolution of early development of the nervous system: a comparison between arthropods. Bioessays 2005, 27:874-883.

7. Janssen R, Eriksson BJ, Budd GE, Akam M, Prpic N-M: Gene expression patterns in an onychophoran reveal that regionalization predates limb segmentation in pan-arthropods. Evol Dev 2010, 12:363-372.

8. Meusemann K, Von Reumont BM, Simon S, Roeding F, Strauss S, Kück P, Ebersberger I, Walzl M, Pass G, Breuers S, Achter V, Von Haeseler A, Burmester T, Hadrys H, Wägele JW, Misof B: A phylogenomic approach to resolve the arthropod tree of life. Mol Biol Evol 2010, 27:2451-2464.

9. Regier JC, Shultz JW, Zwick A, Hussey A, Ball B, Wetzer R, Martin JW, Cunningham CW: Arthropod relationships revealed by phylogenomic analysis of nuclear protein-coding sequences. Nature 2010, 463:1079-1083.

10. Schaeper ND, Pechmann M, Damen WGM, Prpic N-M, Wimmer EA: Evolutionary plasticity of collier function in head development of diverse arthropods. Dev Biol 2010, 344:363-376.

11. Janssen R, Damen WGM, Budd GE: Expression of collier in the premandibular segment of myriapods: support for the traditional Atelocerata concept or a case of convergence? BMC Evol Biol 2011, 11:50.

12. Janssen R, Damen WGM, Budd GE: Gene expression suggests conserved mechanisms patterning the heads of insects and myriapods. Dev Biol 2011, 357:64-72.

13. Rota-Stabelli O, Campbell L, Brinkmann H, Edgecombe GD, Longhorn SJ, Peterson KJ, Pisani D, Philippe H, Telford MJ: A congruent solution to arthropod phylogeny: phylogenomics, microRNAs and morphology support monophyletic Mandibulata. Proc R Soc Lond B 2011, 278:298-306.

14. Sharma PP, Schwager EE, Extavour CG, Giribet G: Evolution of the chelicera: a dachshund domain is retained in the deutocerebral appendage of Opiliones (Arthropoda, Chelicerata). Evol Dev 2012, 14:522-533.

15. Barnett AA, Thomas RJ: The expression of limb gap genes in the mite Archegozetes longisetosus reveals differential patterning mechanisms in chelicerates. Evol Dev 2013, 15:280-292.

16. Dunn CW, Hejnol A, Matus DQ, Pang K, Browne WE, Smith SA, Seaver E, Rouse GW, Obst M, Edgecombe GD, Sørensen MV, Haddock SHD, Schmidt-Rhaesa A, Okusu A, Kristensen RM, Wheeler WC, Martindale MQ Giribet G: Broad phylogenomic sampling improves resolution of the animal tree of life. Nature 2008, 452:745-749.

17. Hejnol A, Obst M, Stamatakis A, Ott M, Rouse GW, Edgecombe GD, Martinez P, Baguñà J, Bailly X, Jondelius U, Wiens M, Müller WEG, Seaver E, Wheeler WC, Martindale MQ, Giribet G, Dunn CW: Assessing the root of bilaterian animals with scalable phylogenomic methods. Proc R Soc Lond B 2009, 276:4261-4270.

18. Panganiban G, Nagy L, Carroll SB: The role of the Distal-less gene in the development and evolution of insect limbs. Curr Biol 1994, 4:671-675.

19. Popadić A, Panganiban G, Abzhanov A, Rusch D, Shear WA, Kaufman TC: Molecular evidence for the gnathobasic derivation of arthropod mandibles and the appendicular origin of the labrum and other structures. Dev Genes Evol 1998, 208:142-150.

20. Scholtz G, Mittmann B, Gerberding M: The pattern of Distal-less expression in the mouthparts of crustaceans, myriapods and insects: new evidence for a gnathobasic mandible and the common origin of Mandibulata. Int J Dev Biol 1998, 42:801-810.

21. Mohler J, Mahaffey JP, Deutsch E, Vani K: Control of Drosophila head segment identity by the bZIP homeotic gene cnc. Development 1995 , 121:237-247.

22. McGinnis N, Ragnhildstveit E, Veraksa A, McGinnis W: A cap ' $n$ ' collar protein isoform contains a selective Hox repressor function. Development 1998, 125:4553-4564.

23. Veraksa A, McGinnis N, Li X, Mohler J, McGinnis W: Cap ' $n$ ' collar B cooperates with a small Maf subunit to specify pharyngeal development and suppress deformed homeotic function in the Drosophila head. Development 2000, 127:4023-4037.

24. Regulski M, McGinnis N, Chadwick R, McGinnis W: Developmental and molecular analysis of Deformed; a homeotic gene controlling Drosophila head development. EMBO J 1987, 6:767-777.

25. Coulcher JF, Telford MJ: Cap'n'collar differentiates the mandible from the maxilla in the beetle Tribolium castaneum. EvoDevo 2012, 3:25.

26. Rogers BT, Peterson MD, Kaufman TC: The development and evolution of insect mouthparts as revealed by the expression patterns of gnathocephalic genes. Evol Dev 2002, 4:96-110.

27. Birkan M, Schaeper ND, Chipman AD: Early patterning and blastodermal fate map of the head in the milkweed bug Oncopeltus fasciatus. Evol Dev 2011, 13:436-447.

28. Rehm EJ, Hannibal RL, Chaw RC, Vargas-Vila MA, Patel NH: Fixation and dissection of Parhyale hawaiensis embryos. CSH Protocols 2009, 2009:pdb.prot5127.

29. Sharma PP, Schwager EE, Extavour CG, Giribet G: Hox gene expression in the harvestman Phalangium opilio reveals divergent patterning of the chelicerate opisthosoma. Evol Dev 2012, 14:450-463.

30. Farley RD: Development of segments and appendages in embryos of the desert scorpion Paruroctonus mesaensis (Scorpiones: Vaejovidae). J Morphol 2001, 250:70-88. 
31. Zeng V, Extavour CG: ASGARD: an open-access database of annotated transcriptomes for emerging model arthropod species. Database 2012, 2012:bas048.

32. Lynch JA, Peel AD, Drechsler A, Averof M, Roth S: EGF signaling and the origin of axial polarity among the insects. Curr Bio/ 2010, 20:1042-1047.

33. Rehm EJ, Hannibal RL, Chaw RC, Vargas-Vila MA, Patel NH: In situ hybridization of labeled RNA probes to fixed Parhyale hawaiensis embryos. CSH Protocols 2009, 2009:pdb.prot5130.

34. Edgar RC: MUSCLE: multiple sequence alignment with high accuracy and high throughput. Nucleic Acids Res 2004, 32:1792-1797.

35. Bowerman B, Eaton BA, Priess JR: skn-1, a maternally expressed gene required to specify the fate of ventral blastomeres in the early $C$. elegans embryo. Cell 1992, 68:1061-1075.

36. Motohashi H, Yamamoto M: Nrf2-Keap1 defines a physiologically important stress response mechanism. Trends Mol Med 2004, 10:549-557.

37. Sykiotis GP, Bohmann D: Stress-activated cap'n'collar transcription factors in aging and human disease. Sci Signal 2010, 3:re3.

38. Misra JR, Horner MA, Lam G, Thummel CS: Transcriptional regulation of xenobiotic detoxification in Drosophila. Genes Dev 2011, 25:1796-1806.

39. Janssen R, Damen WGM: The ten Hox genes of the millipede Glomeris marginata. Dev Genes Evol 2006, 216:451-465.

40. Hughes $\mathrm{CL}$, Kaufman TC: Exploring the myriapod body plan: expression patterns of the ten Hox genes in a centipede. Development 2002, 129:1225-1238.

41. Abzhanov A, Popadic A, Kaufman TC: Chelicerate Hox genes and the homology of arthropod segments. Evol Dev 1999, 1:77-89.

42. Schwager EE, Schoppmeier M, Pechmann M, Damen WGM: Duplicated hox genes in the spider Cupiennius salei. Front Zool 2007, 4:10.

43. Eriksson BJ, Tait NN, Budd GE, Janssen R, Akam M: Head patterning and Hox gene expression in an onychophoran and its implications for the arthropod head problem. Dev Genes Evol 2010, 220:117-122.

44. Schoppmeier M, Damen WGM: Double-stranded RNA interference in the spider Cupiennius salei: the role of Distal-less is evolutionarily conserved in arthropod appendage formation. Dev Genes Evol 2001, 211:76-82.

45. Khila A, Grbic M: Gene silencing in the spider mite Tetranychus urticae dsRNA and siRNA parental silencing of the Distal-less gene. Dev Genes Evol 2007, 217:241-251.

46. Sharma PP, Schwager EE, Giribet G, Jockusch EL, Extavour CG: Distal-less and dachshund pattern both plesiomorphic and apomorphic structures in chelicerates: RNA interference in the harvestman Phalangium opilio (Opiliones). Evol Dev 2013, 15:228-242.

doi:10.1186/2041-9139-5-3

Cite this article as: Sharma et al: Subdivision of arthropod cap-n-collar expression domains is restricted to Mandibulata. EvoDevo 2014 5:3.

\section{Submit your next manuscript to BioMed Central and take full advantage of:}

- Convenient online submission

- Thorough peer review

- No space constraints or color figure charges

- Immediate publication on acceptance

- Inclusion in PubMed, CAS, Scopus and Google Scholar

- Research which is freely available for redistribution 